\title{
Model-Plant Performance Matching from the Limited Number of Measured Quantities for Gas Power Generator
}

\author{
Wang Lifeng \\ Field Bus Technology \& Automation Lab \\ North China University of Technology \\ Beijing,China
}

\author{
Zhong Datian \\ Beijing Aerospace Propulsion Institute \\ Beijing,China
}

\begin{abstract}
The matching technique of model-plant performance data can be used to solve several problems, such as fault diagnosis, the model calibration and deterioration estimation etc. In present paper, model-plant performance matching from the limited number of measured quantities is proposed. A gas power generator is modeling in simulating environments to constitute a benchmark case. Assumed the health factors are constant at adjacent zone of operating points, several discrete points are selected to compensate the number of measured quantities. Depending on the interrelated equations between measured residuals and health factors, the nonlinear Least Square is used to optimize the object value of components health factors. Several sets of simulated deterioration and fault data are naturalized as the testing case, the better matching of model-plant is obtained from the simulating results.
\end{abstract}

Keywords-Engine model;deterioration estimation;fault diagnosis; model-plant matching

\section{INTRODUCTION}

Performance simulation model of gas power generator is playing an important role in design, development and availability. But the much complaint from the customers happens when it is put into use in the application because of the mismatching of model-plant testing data.

It may be caused by three situation, as follows: a) The problem of healthy plant, such as the individual product quality, changes over time of the plant design and manufacturing process, performance differences between part vendors, deterioration of engine during testing and service. b) The problem of modeling, the analysis assumptions of model, un-modeled, imperfectly modeled or incorrectly modeled physical phenomena in engine, c) The faulty plant, the faults of one or more plant components occur during service.

The deviation between model and plant is observed from residuals of the measured quantities and model predicting output. The problem is to seek a solution to determine the values of the tuning health factors from a given series of measured residuals. Least Squares Techniques coupled with Gas Path Analysis(GPA) are introduced in fault detecting and diagnosis by Doe[1,2]. Mathiodakis[3] gives a good overview of the basic gas path assessment problem and discusses some general techniques used in its solution. The nonlinear adaptive modeling techniques are introduced to fine matching of model-plant although it is more sophisticated[4,5]. Multiple discrete points optimization approach is adopt because it can overcome the lack of measurements[6,7]. The adaptive GPA and genetic algorithm is also proposed into matching of model and testing data by $\mathrm{Li}[8,9,10]$. As well as the probabilistic fusion wit GPA derived from diagnosis methods is applied to the model and testing data matching $[11,12]$. The most difficulties faced on model-plant matching are the limited number of measured quantities which related the performance. The solution is based on the augment of measured quantities or the reduction of estimated health factors to ensure that the number of equations is equal or more than the number of estimated factors.

In present paper, model-plant performance matching from the limited number of measured quantities is proposed. A gas power generator is modeling in simulating environments[13] to constitute a benchmark case. Assumed the health factors are constant at adjacent zone of operating points, several discrete points are selected to compensate the number of measured quantities. Depending on the interrelated equations between measured residuals and health factors, the nonlinear Least Square is used to optimize the object value of components health factors. Several sets of simulated deterioration and fault data are naturalized as the testing case, the better matching of model-plant is obtained from the simulating results.

\section{Gas Power Generator Layout}

A gas power generator which is representative used in peak power is selected as a demonstrating object. Refer figure 1, the gas power generator have been modeling in simulation environment. It is a two-spool turbojet with power generator configuration. The five rotating components consist of the Booster (Bst), Power(Pow) generator and low pressure turbine (Lturb) on the booster shaft, the pressure compressor (Com) and high pressure turbine (Hturb) on the core shaft. The hot gas is generated by natural gas burning in main burner(Mburn), which drive the turbine shaft to rotate. The gas is exhausted through the Nozzle(Nozz).

The power generator is small size, operating with an overall pressure ratio of $24,38 \mathrm{~kg}$ air flow, $5 \mathrm{MW}$ power level. The control inputs to this engine are main fuel flow (wfc). Two spool rotation and five gas path sensors are considered as measured quantities for control: N0, N1, P0, 
T0, P3, P5, T5. This is representative of the sensors used in most gas power generator engines.

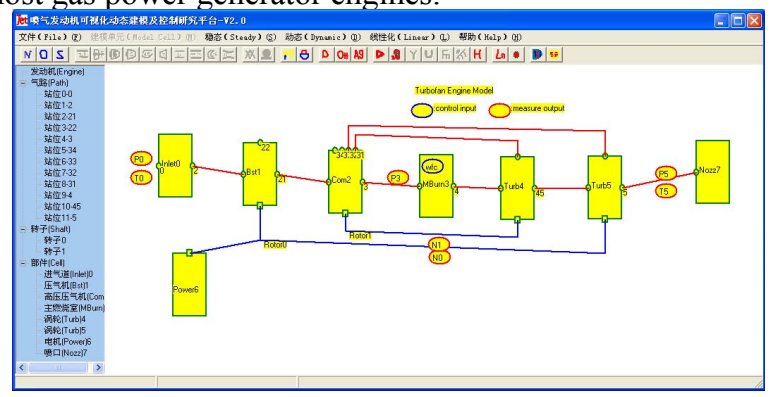

Figure 1. Gas power generator model skeleton in simulation environment

According to the detailed components level thermodynamic model at steady state operating point, the model output of refer to operating point is described bellow:

$$
\mathbf{y}_{r}=\mathbf{y}\left(\mathbf{f}_{r}, \mathbf{u}_{r}\right)
$$

Where $\mathbf{u}_{r}$ is input vector of refer operating point, the steady state operating point of power generator is defined by the control main fuel flow (wfc), Inlet temperature (T0) and pressure (P0). The $\mathbf{y}_{r}$ is output vector of refer engine model, $\mathbf{f}_{r}$ is the health factor vector. The health factor $\mathbf{f}_{r}$ is zero if model-plant match, it will non-zero if mismatching. Theoretically, $\mathbf{f}_{r}$ vary with input of refer operating point $\mathbf{u}_{r}$. But in the adjacent zone of steady state operating points, we can assume that the $\mathbf{f}_{r}$ is constant.

The health parameter employed to characterize the fault of rotating components are an efficiency $\eta$ and flow capability $\omega$. These components include Bst, Com, Hturb and LTurb. But the health parameter of Mburn is burner efficiency $\eta$, and the health parameter of Nozz is flow capability $\omega$. So the listing of health parameter is referring the table 1 .

Table 1: Listing of health parameter for power generator component

The health factor is defined as follows:

$f_{i}=\left(h_{m i}-h_{r i}\right) / h_{r i} \quad \mathrm{i}=0,1, \ldots, 9$

where the $h_{m i}$ is the health parameter, $h_{r i}$ is the health parameter at the refer point.

The set of measurements used for control is follows: N0, $\mathrm{N} 1, \mathrm{P} 3, \mathrm{P} 5, \mathrm{~T} 5$. The listing of measured quantities is referred to Table 2:

Table 2: Listing of measured quantities

The scaled residuals are defined as

$d_{i}=\left(y_{m i}-y_{r i}\right) / y_{r i} \quad \mathrm{i}=0,1, \ldots, 5$

where the residual is the plant measured quantities $y_{m i}$,

$y_{r i}$ is refer point value.
Combining the equation (1),(2)and (3), the interrelated vector equations between measured residuals and health factors can be represented for

$$
\mathbf{d}_{r}=\left(\mathbf{y}_{m}-\mathbf{y}_{r}\right) / \mathbf{y}_{r}=\left(\mathbf{y}_{m}-\mathbf{y}\left(\mathbf{f}, \mathbf{u}_{r}\right)\right) / \mathbf{y}\left(\mathbf{f}, \mathbf{u}_{r}\right)=\mathbf{g}\left(\mathbf{f}, \mathbf{u}_{r}\right) \text { (4) }
$$

In equation (4), there are 10 health factors but only 5 equations are available. If the scaled residuals are knows through measurements, there are infinite number of sets of 10 parameter values satisfying the equations at one refer point. Certainly, the different answers can satisfy the equations under different criterions of solved equations.

\section{Model-Plant Matching By Using SEVERAL ADJACENT OPERATING PoINTS DATA}

Assumed the scale health factors are constant at adjacent zone of operating points, we can choose several discrete operating points to compensate the number of measured quantities. Then the number of measured quantities is more than the number of equations, the unique solution of equations is gained if the equations (4) are nonlinear. If any $\mathrm{q}+1$ adjacent operating points are chosen, that $\mathbf{u}_{r i}$ are given, we can get the equations as follows:

$$
\begin{aligned}
& \mathbf{d}_{r 0}=\mathbf{g}\left(\mathbf{f}, \mathbf{u}_{r 0}\right) \\
& \mathbf{d}_{r 1}=\mathbf{g}\left(\mathbf{f}, \mathbf{u}_{r 1}\right) \\
& \ldots \ldots \\
& \mathbf{d}_{r q}=\mathbf{g}\left(\mathbf{f}, \mathbf{u}_{r q}\right)
\end{aligned}
$$

In equation (5), the interrelated equations between scale measured residuals and health factors is described of several discrete operating points.

$$
\mathbf{d}=\mathbf{g}(\mathbf{f})
$$

In equation (6), the health factors are less than the number of equations, the regularization methods is used to optimize the object value on the health factors of engine components. The optimizing target function is defined as follows:

$$
\min \phi(\mathbf{f})=\left\|\mathbf{g}(\mathbf{f})-\mathbf{d}_{m}\right\|^{2}+\alpha\|\mathbf{f}\|^{2}
$$

$\mathbf{d}_{m}$ is measurement residuals, $\alpha$ is regularization coefficients, $\|\mathbf{f}\|$ is regularization items of health factor, $I$ is unit matrix. In order to gains the $\min \phi(\mathbf{f})$, it is satisfied as follows:

$$
\frac{\partial \phi(\mathbf{f})}{\partial \mathbf{f}}=\mathbf{J}(\mathbf{f})^{T}\left(\mathbf{g}(\mathbf{f})-\mathbf{d}_{m}\right)+\alpha \mathbf{I f}=0
$$

The iteration equation steps of solving equations (8) are as follows:

$$
\begin{aligned}
& \left(\mathbf{J}\left(\mathbf{f}^{k}\right)^{T} \mathbf{J}\left(\mathbf{f}^{k}\right)+\alpha \mathbf{I}\right) \boldsymbol{d} \mathbf{f}=\mathbf{J}\left(\mathbf{f}^{k}\right)^{T}\left(\mathbf{d}_{m}-\mathbf{g}\left(\mathbf{f}^{k}\right)\right)-\alpha \mathbf{I} \mathbf{f}^{k} \\
& \mathbf{f}^{k+1}=\mathbf{f}^{k}+\delta \mathbf{f}
\end{aligned}
$$


Where $\mathbf{J}\left(\mathbf{f}^{k}\right)=\frac{\partial \mathbf{g}(\mathbf{f})}{\partial \mathbf{f}}$ is the Jacobian matrix of function $\mathbf{g}(\mathbf{f})$ at $\mathbf{f}^{k}, \mathbf{J}\left(\mathbf{f}^{k}\right)^{T}$ is the transfer matrix of $\mathbf{J}\left(\mathbf{f}^{k}\right)$, superscript $\mathrm{k}$ is iteration step.

\section{Case Evaluate And Validation}

In order to evaluate the effectiveness of proposed approach applying to model-plant match of power generator, the cases, which cover the fault, calibration and deterioration, are performed compared with combination approach of health factors based on single point data. All measured quantities is simulated from simulation environment and assumed to noise free if averaged with sample time.

\section{Case A: calibration}

The normal performance model stands for the average of all product line, so the deviation between individual plant and the normal model exist because of product quality. For example, at standard atmosphere in ground, the health factors of components distribute refer the figure 2, the scaled residuals distribute refer 3 .

Two adjacent points near the refer point are selected as model-plant matching points. The input $\mathbf{u}_{r 0}$ is refer input, the input $\mathbf{u}_{r 1}$ and $\mathbf{u}_{r 2}$ changes around $\mathbf{u}_{r 0}$. The inlet pressure (P0) and temperature (T0) keep unchanged. At the first point, wfc decrease $0.5 \%$, wfc increase $0.5 \%$.At the second point, wfc decrease $1 \%$, wfc increase $1 \%$. .The optimizing results of health factors $f$ refer to figure 3 . The corresponding scaled residuals distribute refer the figure 4 .

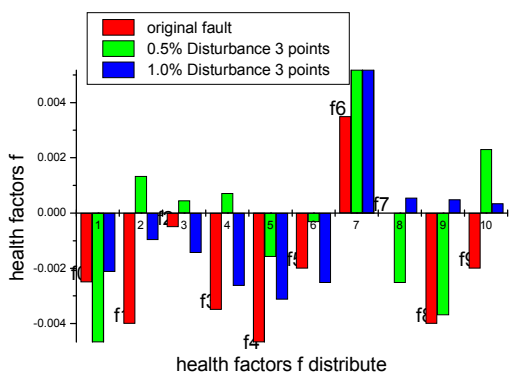

Figure 2. health factors distribute

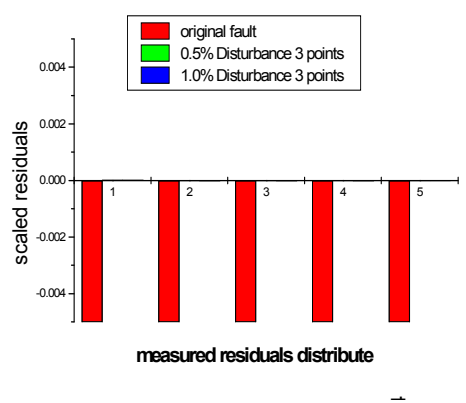

Figure 3. measured residuals distribute
Although the same fine tuning measured residuals of $1 \%$ and 3\% disturbance inputs around refer adjacent points, the better health factor distribute have been gained from the $3 \%$ disturbance inputs. This may be caused by the extent of nonlinear equations at $3 \%$ disturbance inputs.

Case B: deterioration

The deterioration of power generator happens with service cycles. The deterioration level may be higher than effects of loads and rub. In ground, the health factors of components deterioration distribute refer the figure 4 , the scaled residuals distribute refer 5.The margin of health factor is larger than calibration.

Two adjacent points near the refer point are selected as model-plant matching points. The input $\mathbf{u}_{r 0}$ is refer input, the input $\mathbf{u}_{r 1}$ and $\mathbf{u}_{r 2}$ changes is same as case A. Efficiency and flow capability of Bst, Com decrease in scope of $1.5 \%$. But efficiency of Hturb, Lturb decrease, and flow capability increase when deteriorating. The optimizing results of health factors f refer to figure 5 . The corresponding scaled residuals distribute refer the figure 6 .

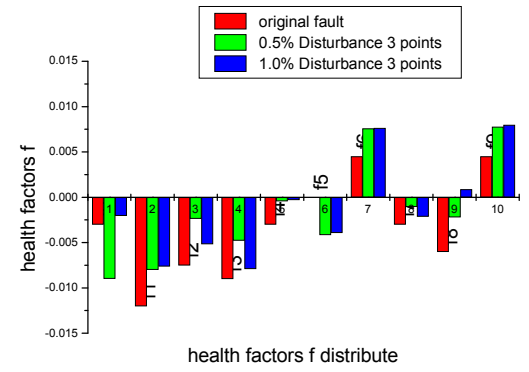

Figure 4. health factors distribute

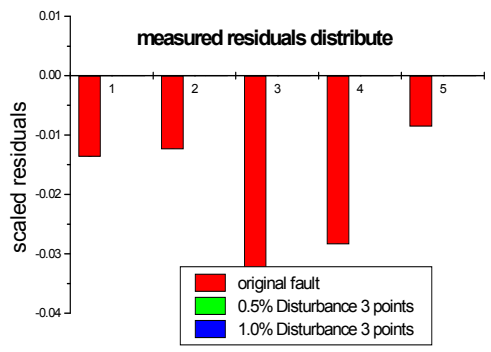

Figure 5. measured residuals distribute

We gain the same tuning measured residuals of $\pm 0.5 \%$, $\pm 1 \%$ disturbance inputs around refer adjacent points, the better health factor distribute have been gained from the $1 \%$ disturbance inputs than the $0.5 \%$.

Case C: fault

Component fault is consequently occurring when it working, but we may not know the location of fault in prior. If we know which component fault occurs, we can reduce the number of optimizing health factors so as to gain the unique solutions. Now assumed that the Com and Mburn have faults, which the efficiency of Com reduced by $1 \%$, flow capability 
reduced by $0.6 \%$, and efficiency of Mburn reduced by $0.5 \%$. The health factors of components distribute refer the figure 7 , the scaled residuals distribute refer 8 .

Two adjacent points near the refer point are selected as model-plant matching points. The input is same as case A, but wfc decrease $1 \%$ at first point, wfc increase $1 \%$ at second point. In the meantime, we can access the fault by using the single points data. Then the optimizing results of health factors $\mathbf{f}$ refer to figure 6 . The corresponding scaled residuals distribute refer the figure 7 .

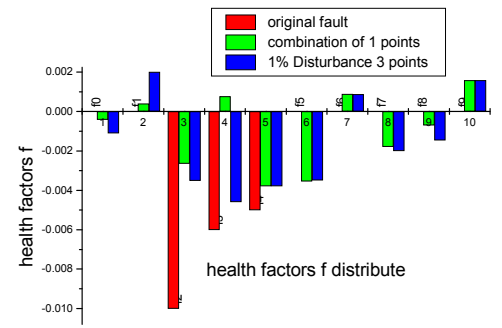

Figure 6. health factors distribute

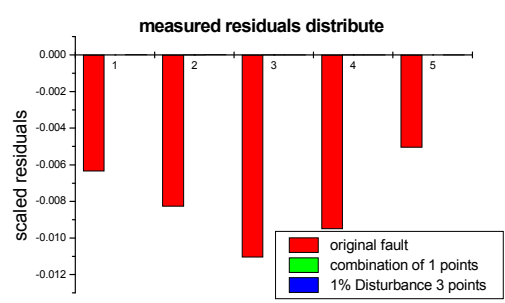

Figure 7. measured residuals distribute

The fine tuning measured residuals were gained by using several points optimization or one point but the f distributes is not very similar each other although the best target is accept. And sometime the calculation collapse due to health factor beyond the reasonable scope.

\section{CONCLUSIONS}

Model-plant performance matching from the limited number of measured quantities is invalidated through calibration, deterioration and fault case optimization of several adjacent operating point data. The better matching of model-plant is obtained from the simulating results. The method is more suitable for model-plant matching at steady operating conditions.

Acknowledge:The work for this paper is sponsored by

Beijing Municipal Education Commission funds (KM200410009006, KM200610009001) \& PHR(IHLB). Many thanks for supporting my works from my friends.6.

\section{REFERENCES}

[1] Doel D. "An assessment of weighted-least-squares based gas path analysis", J. Eng. Gas Turbines Power,April 1994,Volume 116,Issue 2,366

[2] D. L. Doel ;'Interpretation of Weighted-Least-Squares Gas Path Analysis Results" Journal of Engineering for Gas Turbines and Power, JULY 2003, Vol. 125 ,p625

[3] Mathioudakis, K., Kamboukos, P. "Assessment of Gas Path Diagnostic Schemes," ASME Turbo Expo paper GT2003-53862, 2003.

[4] N. Aretakis ; K. Mathioudakis ;'Non-linear engine component fault diagnosis from a limited number of measurements using a combinatorial approach" Proceedings of ASME Turbo Expo 2002 ASME-GT-2002-30031

[5] Stamatis A., Mathioudakis K., Papailiou K. D., 1990, “Adaptive Simulation of Gas Turbine Performance", Journal of Engineering for Gas turbines and Power, April 1990, vol. 112, pp. 168-175

[6] Gulati A., Taylor D., Sign R., 2001, "Multiple operating point analysis using genetic algorithm optimization for gas turbine diagnostic", XV ISABE, Bangalore, India, Sept. 3-7,2001, paper ISABE-2001-1139.

[7] Stamatis A., Mathioudakis K., Berios G., Papailiou K.,1991,: "Jet Engine Fault Detection with Discrete Operating Points Gas Path Analysis", Journal of Propulsion, 7, No 6, Dec 1991, pp 1043-1048.

[8] Y. G. Li, M. F. Abdul Ghafir, L. Wang etc." Nonlinear Multiple Points Gas Turbine Off-Design Performance Adaptation Using a Genetic Algorithm“, J. Eng. Gas Turbines Power -- July 2011 -Volume 133, Issue 7, 071701 (9 pages)

[9] Li, Y. G., Marinai, L., Lo Gatto, E., Pachidis, V., and Pilidis, P., 2009 , "Multiple Point Adaptive Performance Simulation Tuned to Aerospace Test-Bed Data,” J. Propul. Power, 253 , pp. 635-641.

[10] Y. G. Li, "Gas Turbine Performance and Health Status Estimation Using Adaptive Gas Path Analysis", Journal of Engineering for Gas Turbines and Power APRIL 2010, Vol. 132

[11] Roth, B. R., Mavris, D., Doel, D. L., and Beeson, D., 2004, "HighAccuracy Matching of Engine Performance Models to Test Data," ASME Paper No. GT2003-38784.

[12] Roth, B. A., Doel, D. L., and Cissell, J. J., 2005, "Probabilistic Matching of Turbofan Engine Performance Models to Test Data," ASME Paper No. GT2005-68201.

[13] Wang lifeng; "Study on Visual Dynamic Modeling Environment of Turbojet Engine", 12th conference on aerodynamic \& thermodynamic of propulsion system of China, Sichuan, 2009/9.

TABLE I. LISTING OF HEALTH PARAMETER FOR POWER GENERATOR COMPONENT

\begin{tabular}{|c|c|c|c|c|c|c|c|c|c|c|}
\hline Components & Bst & & Cor & & Mburn & Htu & & Ltu & & Nozz \\
\hline Heal th $h_{m i}$ & $\eta$ & $\omega$ & $\eta$ & $\omega$ & $\eta$ & $\eta$ & $\omega$ & $\eta$ & $\omega$ & $\omega$ \\
\hline f act or $f_{i}$ & $f_{0}$ & $f_{1}$ & $f_{2}$ & $f_{3}$ & $f_{4}$ & $f_{5}$ & $f_{6}$ & $f_{7}$ & $f_{8}$ & $f_{9}$ \\
\hline
\end{tabular}


TABLE II. LISTING OF MEASURED QUANTITIES

\begin{tabular}{|l|l|l|}
\hline Measured quantity & measured $y_{m i}$ & scaled residuals $\mathcal{E}_{i}$ \\
\hline Booster shaft speed & N0 & $d_{0}$ \\
\hline Core shaft speed & N1 & $d_{1}$ \\
\hline Compressor Outlet Pressure & P3 & $d_{2}$ \\
\hline LP Turbine Outlet Pressure & P5 & $d_{3}$ \\
\hline LP Turbine Outlet Temperature & T5 & $d_{4}$ \\
\hline
\end{tabular}

